\title{
Los entornos virtuales de aprendizaje (EVA) en la disrupción del proceso enseñanza-aprendizaje
}

\author{
Aranda Yanoc Ángel \\ laranday@ucvvirtual.edu.pe \\ https://orcid.org/0000-0002-8674-4056 \\ Vilchez Bula Elizabeth Rosario \\ evilchez024@gmail.com \\ https://orcid.org/0000-0001-6239-7136
}

\section{RESUMEN}

El entono virtual de aprendizaje (EVA) es un escenario que favorece el aprendizaje de los estudiantes con el apoyo de las herramientas digitales y tecnológicas mediadas de manera oportuna por el docente. En tal sentido el objetivo es explicar el rol de los entornos virtuales en la disrupción de los procesos de enseñanza y aprendizaje en la educación no presencial. Para lograrlo se procedió a la búsqueda de información en los motores de búsqueda: Scielo, Redalyc, Dialnet, Ebesco empleando los filtros necesarios y posteriormente se realizó el procesamiento de la información. Respecto a los resultados la educación ha sufrido un cambio de paradigma, transitando del modelo de "sabio en el escenario" al modelo de "guía al costado" en un entorno educativo. Los enfoques pedagógicos innovadores emergen en torno a los nuevos contextos configurados por el ejercicio de las TIC en educación; además, las nuevas tecnologías se asocian a definiciones, herramientas e innovaciones empleados en los entornos educativos.

Palabras clave: entorno; virtual; disrupción; enseñanza; aprendizaje. 


\title{
Virtual learning environments (VLE) in the disruption of the teaching-learning process
}

\begin{abstract}
The virtual learning environment (VLE) is a scenario that favors student learning with the support of digital and technological tools mediated in a timely manner by the teacher. In this sense, the objective is to explain the role of virtual environments in the disruption of teaching and learning processes in remote education. To achieve this, we proceeded to search for information in search engines: Scielo, Redalyc, Dialnet, Ebesco using the necessary filters and subsequently the information was processed. Regarding the results, education has undergone a paradigm shift, moving from the "wise man on stage" model to the "guide alongside" model in an educational environment. Innovative pedagogical approaches emerge around the new contexts configured by the exercise of ICT in education; Furthermore, new technologies are associated with definitions, tools and innovations used in educational environments.
\end{abstract}

Keywords: environment; virtual; disruption; teaching; learning.

Artículo recibido: 02 noviembre. 2021 Aceptado para publicación: 28 noviembre 2021 Correspondencia: 1aranday@ucvvirtual.edu.pe Conflictos de Interés: Ninguna que declarar 


\section{INTRODUCCIÓN}

El 7 de marzo de 2020, la Organización Mundial de la Salud (OMS), realizó una declaración tras superarse los 100000 casos de COVID-19, aludiendo a la dimensión pandémica e invocando a asumir las medidas de bioseguridad para evitar su propagación. En este marco, todas las actividades humanas han sufrido una transformación con el predominio de la virtualidad. Por ello, la educación ha mutado de la modalidad presencial a la no presencial como lo ha reglamentado el Ministerio de Educación (MINEDU, 2020a). En este escenario configurado por los entornos virtuales de aprendizaje (EVA), surge la necesidad de innovar a través de estrategias adecuadas que motiven y propicien el desarrollo de competencias para fomentar en los estudiantes su capacidad crítica y reflexiva (Cedeño y Murillo, 2019).

El entono virtual de aprendizaje (EVA) es un escenario que favorece el aprendizaje de los estudiantes con el apoyo de las herramientas digitales y tecnológicas mediadas de manera oportuna por el docente; generando una interacción dinámica entre docente y discente. (Contreras-Colmenares y Garcés-Díaz, 2019), por otro lado, Díaz y Castro (2017) en el entorno virtual de aprendizaje se verifica que "el alumno aprende no es simplemente una copia o una reproducción de lo que en ese entorno se le presenta como contenido a aprender" (p.54). En consecuencia, el aprendizaje virtual resulta más que una simple transposición del contenido externo al esquema mental del estudiante, sino producto de un proceso reconstructivo personal del contenido. En consecuencia, los entornos virtuales constituyen extraordinarias oportunidades para potenciar el proceso de la enseñanza aprendizaje en la interacción de los docentes con los estudiantes en términos de flexibilidad, accesibilidad y muchas veces en costos (Rojas, Angoma, Huayta y Pacheco, 2019).

El proceso de enseñanza-aprendizaje, dentro de la sociedad es una actividad que está en lo político e histórico y que en el transcurso del tiempo se ha visto permeado por los cambios vertiginosos asociados a la producción de componentes tecnológicos. En la era digital vigente, los entornos virtuales han reemplazado al modelo presencial y ha permitido que el acceso a contenidos digitales de aprendizaje se realice de manera síncrona y asíncrona, por lo cual las aulas permiten que el aprendizaje ocurra de manera ubicua (Fernández y Ramírez, 2020). 
En referencia a los trabajos previos revisados en el contexto internacional sobre la variable entornos virtuales de aprendizaje (EVA), tenemos a Rocancio (2019) precisó que, la evaluación en EVA, precisa implementar el HEODAR, la minería de datos, FLOE y LORI, para lo cual es preciso curar los contenidos. Asimismo, Morales (2018) sostuvo que, el proceso de adquisición de las competencias Tecnologías de la información y la comunicación (TIC) permite que el hardware y software puedan ser identificados y manipulados adecuadamente. También, Gelabert (2019) precisó que, utilizar las TIC en el proceso enseñanza-aprendizaje, constituyen el pilar de la educación a distancia y educación presencial-virtual. Otro resultado fue el de Bournissen (2017) quien afirmó que resulta indispensable lograr la participación de los actores educativos para el empoderamiento del entorno virtual de aprendizaje.

A nivel nacional, se tiene a Carrera (2019) quien concluyó que, a través de la creación de MI-EVA, permite que el docente mejore su competencia tecnológica. Asimismo, Arévalo (2018), quien sostuvo que, para el logro de aprendizajes esperados en las diversas áreas, es preciso implementar una didáctica desde el entorno virtual.

Por lo expuesto, la presente investigación plantea como objetivo explicar el rol de los entornos virtuales en la disrupción de los procesos de enseñanza y aprendizaje en la educación no presencial. Permitiendo que todos los procesos implicados en este proceso se adapten a los nuevos entornos y postulados del nuevo ciudadano en una sociedad del conocimiento que asocia de manera armónica la realidad con la virtualidad.

Aparicio-Gómez y Ostos-Ortiz (2021), precisan que las pedagogías emergentes hacen su aparición en el escenario de los ambientes virtuales de aprendizaje en tiempos de gran incertidumbre. Emergiendo de manera disruptiva en el modo de planificar y desarrollar el proceso educativo centrado en el aprendizaje. Así como lo establece el MINEDU (2020b), fundamentando la preparación de la enseñanza orientada para el aprendizaje de los estudiantes. Esta visión motiva una disrupción en el paradigma relacionado al rol del profesional docente, quien es concebido como mediador estratégico de los aprendizajes.

\section{MATERIALES Y MÉTODOS}

El artículo de revisión selecciona de manera crítica y reflexiva la bibliografía publicada, en relación con un tema preciso y que resulte de interés para la comunidad de investigadores; para ello se le debe brindar una perspectiva innovadora acorde a los cambios verificados (Reyes, 2020). Por lo mencionado, se realizó la revisión de la 
bibliografía, previamente seleccionada, publicada en los últimos años de las tesis, los artículos científicos y de revisión publicadas en los repositorios y las revistas indexadas, analizando y destacando las propuestas manifestadas en ellas.

Se realizó la búsqueda de información en la base de datos Ebesco, Scielo, Dialnet y Redalyc, comprendido en el periodo de marzo del año 2017 a setiembre de 2021; empleando los términos de búsqueda: entorno virtual de aprendizaje, ambiente virtual de aprendizaje, aprendizaje a distancia. Con el propósito de ubicar la información de los artículos destacados y con información reciente. Lo cual nos permitió analizar la información relacionada a los entornos virtuales de aprendizaje y los ambientes virtuales de aprendizaje. Se procedió en secuencia, identificando el título, el resumen, las palabras clave los resultados y la conclusión.

Para la búsqueda de fuentes de información confiables se emplearon los siguientes filtros: el idioma para diferenciar los artículos publicados en inglés y español; el tipo de publicación para establecer la relación con nuestro artículo; la fecha de publicación para establecer la actualidad de la información; el área de investigación para limitar la búsqueda a educación y la accesibilidad para establecer la libertad del acceso.

Para realizar el proceso de análisis de las fuentes acopiadas, se procedió a ingresar la información a una matriz elaborada para tal fin, en la cual se consignan los siguientes datos: información acerca del autor, el nombre de la revista indexada, el año de publicación, la cita textual asumida, el parafraseo de la cita y la referencia bibliográfica. Todo ello acorde a las normas APA de la 7ma edición.

\section{RESULTADOS Y DISCUSIÓN}

\section{La disrupción digital en el aprendizaje}

Los entonos virtuales para el aprendizaje configuran una etapa nueva; es así como verificamos el proceso evolutivo del aprendizaje 1.0 al aprendizaje 4.0, acorde a los cambios sustanciales en las diversas actividades socioeconómicas que realiza el hombre y corresponde a la evolución acelerada de la Web (Demartini y Benussi, 2017).

La educación ha sufrido un cambio de paradigma, transitando del modelo de "sabio en el escenario" al modelo de "guía al costado" en un entorno educativo. Es así como de una educación 1.0 en el cual se produce la dicotomía de una persona que conoce y la otra no, por lo cual es depositario de información, se llega a la educación 2.0 en el cual el docente cambia su rol de consejero a guía e intermediario. En la educación 3.0 el docente asume 
el rol de liderazgo de creaciones compartidas, cooperativas y colaborativas del conocimiento; rol que se renueva en la educación 4.0, el docente asume un rol mediador del aprendizaje que recurre a la tecnología y las herramientas digitales, basadas en la inteligencia artificial, integradas en los entornos virtuales de aprendizaje - EVA (Brailas, Koskinas, y Alexias, 2017).

Los enfoques pedagógicos innovadores emergen en torno a los nuevos contextos configurados por el uso de las TIC en educación; además, las tecnologías emergentes se asocian a conceptos, herramientas e innovaciones empleados en los entornos educativos (Moni, Mahmud, High, \& Carbajales-Dale, 2020). Por estas razones, las pedagogías emergentes permiten que los estudiantes fortalezcan su aprendizaje apoyándose en las tecnologías y recursos educativos abiertos, mediante los cuales pueden acceder a ingentes volúmenes de información de manera permanente (Aparicio-Gómez y Ostos-Ortiz, 2021).

Los recursos educativos abiertos permiten la generación de oportunidades de aprendizaje continuamente y promueven el pensamiento crítico, creativo y la autonomía del aprendizaje centralizado en el estudiante (Kim, Lee, Leite, \& Huggins-Manley, 2020; Minedu, 2020b). En este nuevo entorno cambia vertiginosamente la manera en la que se generan, distribuyen, acceden y visualizan los datos; por lo cual, los usuarios se desarraigan de su rol de meros receptores pasivos de información. Ahora se asume un rol activo que permite enfocarse en la exploración de datos para una adecuada administración, adquisición, análisis, inferencia y planificación. Por esta razón los estudiantes requieren aprender a trabajar y pensar con datos (Liang \& Hainan, 2019; Yuan, 2019).

En los entornos virtuales de aprendizaje se generan espacios que permiten una fluida comunicación de los usuarios y el acceso a diversos materiales y recursos; caracterizado por la interactividad, flexibilidad, escalabilidad y ubicuidad respecto al aprendizaje. Facilitan la motivación y evaluación (Isela y Otuyemi, 2020). El aprendizaje se potencia cuando se establece una red de conexiones horizontal entre los usuarios, además entre la comunidad de aprendizaje y los recursos que están a disposición de manera libre (Lötter \& Jacobs, 2020).

\section{Innovación de la metodología del aprendizaje}

El entono virtual de aprendizaje exige la implementación de metodologías activas desde 
la concepción curricular centrada en el aprendizaje y en las actividades de autorregulación del estudiante, propiciando su autonomía del aprendizaje con el fin de profundizar el conocimiento y evidenciar el desarrollo de sus competencias (Barroso-Osuna, GutiérrezCastillo, Llorente-Cejudo, \& Ortiz, 2019; Parra-González, Belmonte, Segura-Robles, \& Cabrera, 2020). Existe una diversidad de propuestas metodológicas, siendo las más recurrentes en su empleo el Design thinking que nos ayuda a solucionar problemas, Teachback nos enseña que a través de la conversación se puede aprender, Flipped learning para el acompañamiento y la autonomía y Gamification con la cual se aprende a través de juegos (Aparicio-Gómez y Ostos-Ortiz, 2021).

El Design thinking permite desarrollar la creatividad y el pensamiento crítico al proponer episodios que reta a los estudiantes a resolver una serie de retos que exigen análisis de la situación y alternativas de solución. Para lo cual tiene a disposición diversos recursos y materiales que permite al usuario enfrentar nuevos contextos y situaciones de aprendizaje. Fomenta la innovación y resolución de problemas reales contextualizadas (Withell \& Haigh, 2018). Mientras para el Teachback, el diálogo es clave; pues un experto transmite información, el novato intenta explicar con sus palabras lo que ha entendido y el experto retroalimenta de acuerdo con lo observado.

El aprendizaje invertido transforma el aprendizaje en el aula; pues las competencias más sencillas como memorizar y comprender lo proyecta hacia los entornos habituales de interacción humana del estudiante; mientras que en el aula despliega las competencias más complejas como es aplicar, analizar, evaluar y crear. El Flipped learning potencia la autonomía del aprendizaje en el estudiante; pues considera sus necesidades, estilos y ritmos de aprendizaje en sus escenarios habituales. En el entorno virtual de aprendizaje los materiales y recursos se aprovechan de manera eficiente porque se encuentran a disposición de los usuarios de manera permanente para el logro de los aprendizajes esperados, con una adecuada gestión del tiempo y el trabajo colaborativo asíncrono (Xiu \& Thompson, 2020).

En los entornos virtuales, la masificación de los juegos ha generado que se transformen en un aspecto recurrente en muchos niños y jóvenes, y en este contexto se produce la gamification como una metodología que pretende generar aprendizajes a través de juegos. La estructuración de un juego implica historias extensas, complejas y difíciles, por lo cual los jugadores se obligan a persistir, y cuanto más complejo se torna en un desafío que 
motiva al jugador a continuar para lograr los niveles sucesivos. Jugar mejora la capacidad de resolver problemas, lo cual se estimula a través de recompensas satisfactorias que cumplen el rol de retroalimentación. Cuando el juego se inserta en el entorno virtual de aprendizaje se fomenta la creatividad, el trabajo en equipo colaborativo y la resolución de problemas (Panagiotarou, Stamatiou, Pierrakeas, \& Kameas, 2020).

Los entornos virtuales de aprendizaje permiten que se pueda implementar de manera adecuada la evaluación auténtica o la evaluación formativa, considerando las analíticas de aprendizaje (Aparicio-Gómez y Ostos-Ortiz, 2021). Por ello la irrupción de los entonos virtuales de aprendizaje se considera como un soporte en los procesos de evaluación; en este contexto es necesario vincular la planificación y la evaluación del aprendizaje. En este entorno se puede verificar el progreso de los aprendizajes de cada estudiante, permitiendo recopilar, analizar e interpretar los datos con el fin de tomar decisiones respecto al proceso de aprendizaje individual, generando acciones de retroalimentación. Se configura la ruta de aprendizaje de cada estudiante con el propósito de apoyar el logro de los aprendizajes (Avila, Baldiris, Fabregat, \& Graf, 2020; MINEDU, 2020b).

\section{CONCLUSIÓN}

Los entornos virtuales de aprendizaje (EVA) constituyen extraordinarias oportunidades para potenciar el proceso de la enseñanza -aprendizaje en la interacción de los docentes con los estudiantes en términos de flexibilidad y accesibilidad; por ello se produce una disrupción de las pedagogías que, de manera innovadora, permiten la gestión del conocimiento permitiendo que el estudiante participe de manera autónoma en el recorrido de la ruta de su aprendizaje, empleando los recursos y materiales disponibles en el entorno virtual. En este entorno surgen las pedagogías emergentes, que instauran un diálogo con las tecnologías emergentes y las propuestas psicopedagógicas contemporáneas; pero solo es posible cuando los, docentes y estudiantes asumen prácticas innovadoras, disruptivas como respuesta a las exigencias de la sociedad del conocimiento.

\section{REFERENCIAS BIBLIOGRÁFICAS}

Aparicio-Gómez, O. y Ostos-Ortiz, O. (2021). Pedagogías emergentes en ambientes virtuales de aprendizaje. Revista internacional de pedagogía e innovación educativa, 1(1), 11-36. https://doi.org/10.51660/ripie.v1i1.25

Arevalo, J. (2018). Modelo didáctico para contribuir a la mejora de procesos de enseñanza - aprendizaje en entornos virtuales en la Universidad Señor de Sipán 
modalidad a Distancia en la Región Lambayeque. (Tesis doctoral, Universidad César Vallejo). https://repositorio.ucv.edu.pe/bitstream/handle/20.500.12692/30085/Arevalo_AJ .pdf? sequence $=1 \&$ isAllowed $=y$

Avila, C., Baldiris, S., Fabregat, R. \& Graf, S. (2020). Evaluación de una herramienta de análisis de aprendizaje para apoyar a los docentes en la creación y evaluación de recursos educativos abiertos accesibles y de calidad. BERA, 51(4), 1019-1038. https://doi.org/10.1111/bjet.12940

Barroso-Osuna, J., Gutiérrez-Castillo, J. J., Llorente-Cejudo, M. d. C., \& Valencia Ortiz, R. (2019). Difficulties in the Incorporation of Augmented Reality in University Education: Visions from the Experts. Journal of New Approaches in Educational Research, 8(2), 126-141. https://doi.org/10.7821/naer.2019.7.409

Bournissen, J. (2017). modelo pedagógico para la facultad de estudios virtuales de la Universidad Adventista del Plata. (Tesis doctoral, Universitat de les Illes Balears) https://www.tesisenred.net/bitstream/handle/10803/402708/tjmb1de\%206.pdf?se quence $=1 \&$ is Allowed $=\mathrm{y}$

Brailas, A., Koskinas, K., \& Alexias, G. (2017). Enseñar para emerger: hacia una pedagogía de abajo hacia arriba. Cogent Education, 4(1), 1-14. https://doi.org/10.1080/2331186X.2017.1377506

Carrera, E. (2019). Entornos virtuales de aprendizaje mediante una nueva metodología aplicados a las competencias tecnológicas del docente universitario de la Facultad de Derecho en la Universidad de San Martín de Porres. (Tesis doctoral, Universidad Nacional Federico Villareal). http://repositorio.unfv.edu.pe/bitstream/handle/UNFV/3293/CARRERA\%20SA LAS\%20ERNESTO\%20ADOLFO\%20DOCTOR.pdf?sequence $=1 \&$ is Allowed $=\mathrm{y}$

Cedeño, E. y Murillo, J. (2019). Entornos virtuales de aprendizaje y su rol innovador en el proceso de enseñanza. Rehuso, 4(1), 119-127. https://revistas.utm.edu.ec/index.php/Rehuso/article/view/2156/2275

Contreras-Colmenares, A. y Garcés-Díaz, L. (2019). Ambientes Virtuales de Aprendizaje. Dificultades de uso en los estudiantes de cuarto grado de Primaria. Prospectiva. Revista de Trabajo Social e intervención social, 27, 215-240. 
https://revistaprospectiva.univalle.edu.co/index.php/prospectiva/article/view/727 $\underline{3 / 9783}$

Demartini, C. y Benussi, L. (2017). ). ¿La Web 4.0 y la Industria 4.0 implican Educación X.0? IT Professional, 19(3), 4-7. DOI: 10.1109/MITP.2017.47

Díaz, F., y Castro, A. (2017). Requerimientos pedagógicos para un ambiente virtual de aprendizaje. Cofin Habana, 11(1), 13. http://scielo.sld.cu/scielo.php?script=sci_arttext\&pid=S2073$\underline{60612017000100004 \& \operatorname{lng}=\mathrm{es} \& \operatorname{tlng}=\mathrm{es}}$

Gelabert, J. (2019). Entornos personales de aprendizaje en el desarrollo profesional de los profesores universitarios. (Tesis doctoral, Universitat de les Illes Balears) https://www.tdx.cat/bitstream/handle/10803/671471/tjmgc1de1.pdf?sequence=1

Isela, L. y Otuyemi, E. (2020). Análisis documental: importancia de los entornos virtuales en los procesos educativos en el nivel superior. Revista Tecnología, Ciencia y Educación, (17), 57-77. https://doi.org/10.51302/tce.2020.485

Kim, D., Lee, Y., Leite, W. L., \& Huggins-Manley, A. (2020). Explorar los patrones de uso de estudiantes y maestros asociados con la deserción de estudiantes en una plataforma de aprendizaje en línea abierta respaldada por recursos educativos. Computers and Education, 156. https://doi.org/10.1016/j.compedu.2020.103961

Liang, Q., \& Hainan, N. C. (2019). Adaptive Learning Model and Implementation Based on Big Data. 2019 2nd International Conference on Artificial Intelligence and Big Data, ICAIBD 2019, 183-186. https://doi.org/10.1109/ICAIBD.2019.8836984

Lötter, M. \& Jacobs, L. (2020). Using smartphones as a social constructivist pedagogical tool for inquiry-supported problem-solving: an exploratory study. Journal of Teaching in Travel and Tourism. https://doi.org/10.1080/15313220.2020.1715323

MINEDU (2020a). Orientaciones para el desarrollo del servicio educativo en los centros de educación técnico-productiva e institutos y escuelas de educación superior, en el marco de la emergencia sanitaria por Covid-19. MINEDU. https://cdn.www.gob.pe/uploads/document/file/574851/RVM_N_087-2020MINEDU 1_.PDF

MINEDU (2020b). Diseño Curricular Básico Nacional de la Formación Inicial docente - Programa de estudios de Educación Secundaria especialidad de Comunicación. 
MINEDU. $\quad$ http://www.minedu.gob.pe/superiorpedagogica/producto/disenocurricular-basico-nacional-de-la-formacion-inicial-docente-programa-deestudios-de-educacion-secundaria-especialidad-comunicacion/

Moni, S., Mahmud, R., High, K., y Carbajales-Dale, M. (2020). Evaluación del ciclo de vida de las tecnologías emergentes: una revisión. Journal of Industrial Ecology, 24(1), 52-63. https://doi.org/10.1111/jiec.12965

Morales, Y. (2018). Modelo teórico de un entorno virtual con base en las representaciones sociales de directivos y docentes acerca de las TIC. (Tesis doctoral, Universidad Católica Andrés Bello). http://biblioteca2.ucab.edu.ve/anexos/biblioteca/marc/texto/AAT1231.pdf

Organización Mundial de la Salud (7 de marzo, 2020). Declaración de la OMS tras superarse los $100 \quad 000$ casos de COVID-19. OMS. https://www.who.int/es/news/item/07-03-2020-who-statement-on-cases-ofcovid-19-surpassing-100-000

Panagiotarou, A., Stamatiou, Y., Pierrakeas, C., \& Kameas, A. (2020). Gamification acceptance for learners with different E-skills. International Journal of Learning, Teaching and Educational Research, 19(2), 263-278. https://doi.org/10.26803/IJLTER.19.2.16

Parra-González, M. E., Belmonte, J. L., Segura-Robles, A., \& Cabrera, A. F. (2020). Active and emerging methodologies for ubiquitous education: Potentials of flipped learning and gamification. Sustainability (Switzerland), 12(2). https://doi.org/10.3390/su12020602

Ramírez, L. y Fernández, J. (2020). Entornos virtuales de aprendizaje: usabilidad y alcance en la formación de competencias profesionales del área educativa. Revista Digital FILHA. 15(22), 1-26. http://www.filha.com.mx/publicaciones/edicion/2019-12/entornos-virtuales-deaprendizaje-usabilidad-y-alcance-en-la-formacion-de-competenciasprofesionales-del-area-educativa-por-leticia-nayeli-ramirez-ramirez-y-javierfernandez-de-castro

Reyes, H. (2020). Artículos de Revisión. Revista Médica Chile, 148, 103-108. https://web.archive.org/web/20200506191453id_https://scielo.conicyt.cl/pdf/rm c/v148n1/0717-6163-rmc-148-01-0103.pdf 
Roncancio, C. (2019). Evaluación de los entornos virtuales de enseñanza aprendizaje (EVEA) de la Universidad Santo Tomás Bucaramanga (Colombia) mediante la adaptación y aplicación del sistema Learning Object Review Instrument (LORI). (Tesis docotral, Universitat de les Illes Balears). https://www.tesisenred.net/bitstream/handle/10803/671465/tcyrb1de1.pdf?seque nce $=1 \&$ isAllowed $=y$

Rojas, J., Angoma, M., Huayta, F. y Pacheco, L. (2019). Entornos Virtuales y Aprendizaje de los estudiantes de la Escuela Profesional de Ingeniería de Sistemas de la Universidad Nacional de Huancavelica, Sede Pampas. Apunt. Cienc. Soc. 09(01), 1-7. Rojas, Astucuri, Huayta y Pacheco, 2019) https://journals.continental.edu.pe/index.php/apuntes/article/view/711/670

Yuan, L. (2019). Discussion on the application of computer big data in internet learning. Proceedings - 2019 12th International Conference on Intelligent Computation Technology and Automation, ICICTA 2019, 482-486. https://doi.org/10.1109/ICICTA49267.2019.00109

Withell, A., \& Haigh, N. (2018). Enhancing curricula and learning environments using critical realist perspectives and approaches: a case study and critique. The $\begin{array}{lll}\text { Curriculum 319-337. } & \text { 29(3), Journal, }\end{array}$ https://doi.org/10.1080/09585176.2017.1400451

Xiu, Y., \& Thompson, P. (2020). Flipped university class: A study of motivation and learning. Journal of Information Technology Education: Research, 19, 41-63. https://doi.org/10.28945/4500 